\title{
Investigation of Some Physical Properties of Accretion Induced Collapse in Producing Millisecond Pulsars
}

\author{
Ali Taani ${ }^{1}$ and ChengMin Zhang $^{1}$ \\ National Astronomical Observatories, Chinese Academy of Sciences, Beijing 100012, China \\ alitaani@bao.ac.cn \\ Mashhoor Al-Wardat ${ }^{2}$ \\ Department of Physics, Al-Hussein Bin Talal University, P.O.Box 20, 71111, Ma'an, Jordan \\ and \\ YongHeng Zhao ${ }^{1}$ \\ National Astronomical Observatories, Chinese Academy of Sciences, Beijing 100012, China
}

\begin{abstract}
We investigate some physical characteristics of Millisecond Pulsar (MSP) such as magnetic fields, spin periods and masses, that are produced by Accretion Induced Collapse (AIC) of an accreting white dwarf (WD) in stellar binary systems. We also investigate the changes of these characteristics during the mass-transfer phase of the system in its way to become a MSP. Our approach allows us to follow the changes in magnetic fields and spin periods during the conversion of WDs to MSPs via AIC process. We focus our attention mainly on the massive binary WDs $\left(M \gtrsim 1.0 M_{\odot}\right)$ forming cataclysmic variables, that could potentially evolve to reach Chandrasekhar limit, thereafter they collapse and become MSPs. Knowledge about these parameters might be useful for further modeling of the observed features of AIC.
\end{abstract}

Subject headings: Stars: neutron stars, white dwarfs, cataclysmic variables, x-ray binaries, fundamental parameters

\section{Introduction}

Cataclysmic variables (CVs) are short-period binary systems consisting of massive white dwarfs (WDs) primary that is accreting material via Roche-lobe overflow from low-mass secondary stars (see i. e. Warner (1995); Warner and Woudt (2002); Middleton et al. (2011)), so Accretion Induced Collapse (AIC) is favorable in these systems (van den Heuvel (2004)). However, the AIC scenario has been proposed as an alterna-

\footnotetext{
${ }^{1}$ National Astronomical Observatories, Chinese Academy of Sciences, Beijing 100012, China

${ }^{2}$ Sabbatical visitor, Physics Department, Yarmouk University, P.O.B. 566 Irbid, 21163 Jordan
}

tive source of recycled pulsars sufficient to obviate the difficulties with the standard model (Bhattacharva and van den Heuvel (1991); Hurlev et al. (2010); Taani et al. (2012)). While other authors questioned the viability of an AIC origin for the recycled pulsars on theoretical and statistical grounds (Romani (1990); van den Heuvel (1995)), Ferrario and Wickramasinghe (2008a, $\mathrm{b})$ argued that the AIC channel can form binary Millisecond Pulsars (MSPs) of all observed types with $B \sim 10^{8-9} \mathrm{G}, P \sim 20 \mathrm{~ms}$ and $e \sim 0.44$. (e.g. Bhattacharva and van den Heuvel (1991); Lorimer (2008); Wang et al. (2011)). The required conditions for the formation of an AIC in the case of a steady accretion were summarized in 
Nomoto (1984, 1987), Isern and Hernanz (1994) and in two recent works Chen et al. (2011) and Taani et al. (2012). However, unless the accretion rates are very high $\left(>10^{-7} \mathrm{M}_{\odot} \mathrm{yr}^{-1}\right)$, the accreted (Hydrogen) matter will not go into steady nuclear burning on the surface of the WD, and hence its mass will never grow. In the situation of lower accretion rates, the accreted matter will burn in Super Novae (SN) type Ia, such that the WD will never reach Chandrasekhar limit $\left(1.44 \mathrm{M}_{\odot}\right)$.

Nowadays, there is an increasing amount of evidence which points to the fact that at least a fraction of low-mass X-ray binaries (LMXBs) should be the result of AIC of a WD in a low-mass binary system (Taam and van den Heuvel (1986); Li (2010)). However, this straightforward hypothesis had to face several problems, some of which are not yet completely solved. The behavior of the external layers of an accreting WD depends basically on the rate of mass transfer and on the chemical composition of the accreted material. Even though a lot of work has been done on this issue, much of it is based on very restrictive assumptions (steady spherical accretion, mass for instance).

In general, accretion of material lighter than carbon will induce its burning. Only steady burning or non-disruptive flashing regimes can be allowed in NS formation scenarios (Wickramasinghe and Ferrario 2005). For which conditions this actually happens is still largely undetermined, but it seems that there are several combinations of the parameters mentioned above which allow the star to retain the accreted material and to grow in mass. Even though the WD manages to accrete mass steadily, the subsequent collapse is not yet guaranteed because explosive thermonuclear burning always happens in the contracting core before reaching Chandrasekhar limit. The burning might propagate through the entire star, releasing enough energy to blow it apart (Wickramasinghe et al. 2009).

Observable parameters of binary MSPs, e.g. mass of the pulsar, mass of the companion, spin period of the pulsar, orbital period, orbital eccentricity, etc., are used to probe the past accretion history of the MSPs.

AIC produces WDs having mass less than Chandrasekhar limit, because at least $\sim 10 \%$ of the mass accreted by the WD goes to the binding energy of the nascent NS (Bagchi (2011)); this mechanism is similar to LMXB. While long orbital periods could potentially occur via explosion power from supernova type Ia, which, circumstances has no observational counterparts, consequently, create single MSP. It is currently unknown whether such AIC process appears and how much it contributes to form MSPs population, this is a focus of much present-day research.

The purpose of this paper is to demonstrate how to infer some of the observable quantities and relate them to the dipolar magnetic magnetic field at the surface of the NS, bottom field strength (when the magnetospheric radius $R_{M}=\mathrm{R}$ ) and spin period, during the AIC of a WD in its way to become a member of MSP family.

To accomplish this goal, the model developed by Zhang et al. (2009) is extended to include the magnetic fields and radii for cataclysmic variables (CVs) observed sample. Thus a significant reduction of the polar field strength could occur if such a system accumulates the required critical mass $M_{c r i t}$. Thus we demonstrate the observed properties of the magnetic CVs, that can generally be explained by the model where the field is at most only partially restructured due to accretion.

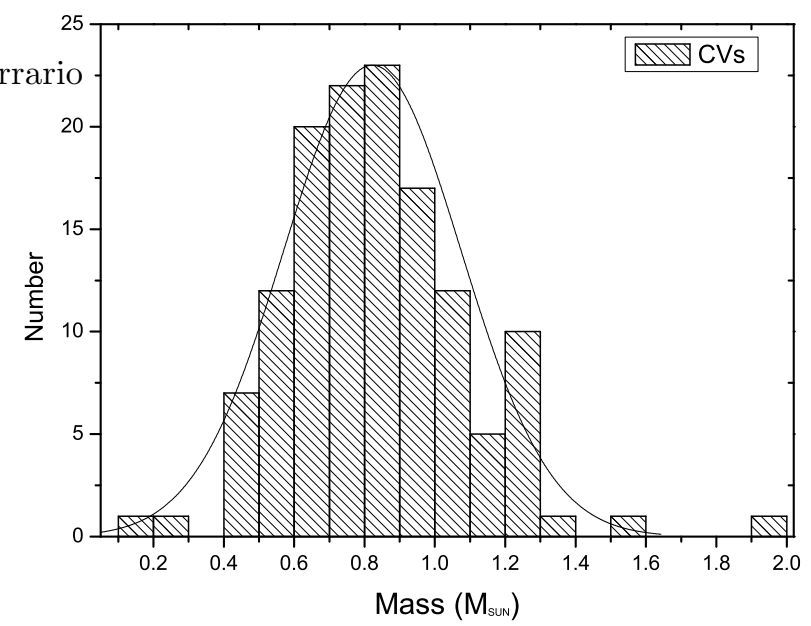

Fig. 1.- Mass distribution of all CVs observed sample. The solid line is the curve fitted using a Gaussian function. The data are taken from Ritter \& Kolb (2011) to construct this distribution. 


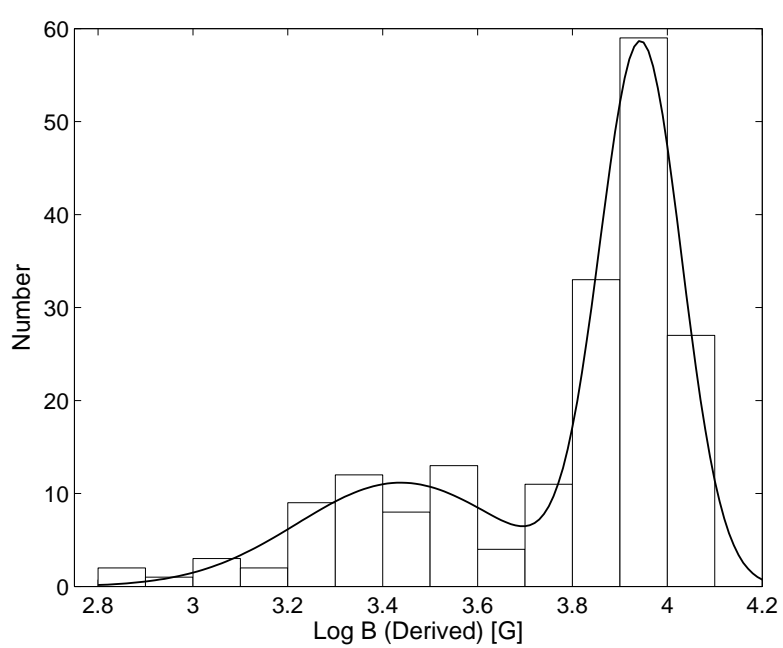

Fig. 2.- Histograms of the bottom magnetic field in CVs. The distribution is relatively Gaussian. Here the field strength of most CVs has been inferred on assumption that CVs of non-spherical accretion on to magnetized NS with a centered dipolar field (see Zhang et al. (2009)). The solid line is the curve fitted using a Gaussian function.

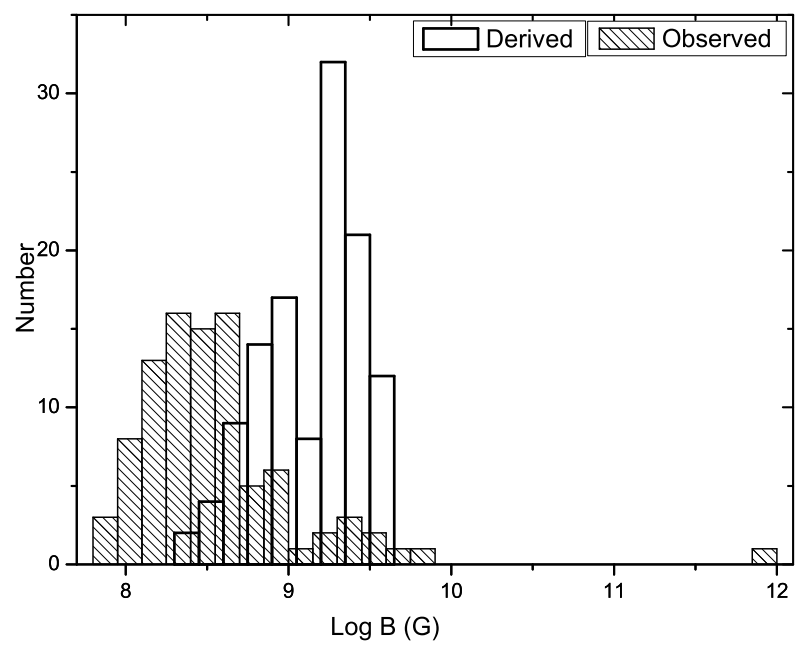

Fig. 3.- Observed magnetic field distributions of MSPs (shaded histogram) and derived ones of the CVs (solid histogram), which were calculated by assuming the conservation of the magnetic flux (see the text). The data for MSPs were taken from the ATNF catalogue (Manchester et al. 2005).

\section{Methodology}

\subsection{The Observed Population of CVs}

Most of the behavior of CVs can be understood in the context of a close binary with mass transfer from a low-mass, near-main-sequence companion star. The mass donor is known as the secondary, and the star which accretes mass is known as the primary. In CVs the primary is a WD, and the secondary is usually a late-type roughly main-sequence star. Interacting binaries are interesting and important to observe, as they allow the study of accretion on observable timescales. Moreover, much can be learnt about binary evolution from them, in particular the effects of accretion on their ultimate evolution. It is predicted that above the $2-3 \mathrm{hr}$ orbital period gap, angular momentum losses are driven by magnetic braking (Ivanova and Taam (2003)), while below the gap the systems evolve via the emission of gravitational wave radiation (see i.e. Spruit and Ritter (1983); Knigge et al. (2011)). However, the theoretical relationship between the mass and radius of a WD is essential in interpreting some observational results. M-R relation was first defined by Chandrasekhar (1939) as:

$$
R \propto \frac{1}{M^{1 / 3}}
$$

for Sirius $\mathrm{B}\left(\mathrm{M}=0.986 \mathrm{M}_{\odot}, \quad \mathrm{R}=0.00846 \mathrm{R}_{\odot}\right)$, where mass and radius are measured in standard solar radii and masses (Wood (1995)). We use this $\mathrm{M}-\mathrm{R}$ relation to derive the radii of $\mathrm{CVs}$ which are not yet observed. The results of the calculated $\mathrm{M}-\mathrm{R}$ values for massive CVs are listed in Table 1.

The sample of mass of CVs we have considered is the set of binary systems collected by Ritter and Kolb (2011) from several high-quality surveys. The sample consists then of 133 systems. Fig. 1 shows the Gaussian distribution of mass of CVs, with mean at $\mathrm{M}_{\mathrm{CV}} \sim 0.822 \mathrm{M}_{\odot}$, where the median mass of these sources is $\mathrm{M}_{\mathrm{CV}}=0.81 \pm 0.16 \mathrm{M}_{\odot}$. Since the Ritter and Kolb (2011) catalog gives a mass of $\mathrm{CH}$ UMa $\mathrm{M}_{\mathrm{CV}} \sim 1.9 \pm 0.30 \mathrm{M} \odot$ (well above the Chandraskhar mass limit for a WD), we decided to exclude this source from our study. We fit the Gauss function to the mass distributions. 
The Gauss function we choose reads,

$$
y=y_{0}+\frac{A}{w_{0} \sqrt{\frac{\pi}{2}}} \exp \left(-2\left(\frac{x-x_{0}}{w_{0}}\right)^{2}\right)
$$

where $y_{0}, x_{0}, w_{0}$ and $A$ are offset of y-axis, center of $\mathrm{x}$-axis, width and area represented by the curve respectively. The fitting results are listed in Table 2.

\subsection{Magnetic field}

The strength of the magnetic field influences the physical conditions in the accretion column, and as a consequence the dominate radiation mechanisms and the evolutionary phase of CVs population (see i.e. Spruit and Ritter (1983); King et al. (1994); Rappaport et al. (1983); Qian et al. (2011)). To derive the bottom magnetic field in CVs (where the field is at most only partially restructured due to accretion), we follow Zhang et al. (2009) model, which suggest that the bottom filed $B_{f}$ for WDs is reached when the magnetospheric radius $R_{\mathrm{M}}=R$, giving

$$
B_{f, W D}=2.8 \times 10^{3} \dot{M}_{16}{ }^{1 / 2} m^{1 / 4} R_{9}^{-5 / 4} \phi^{-7 / 4}
$$

where, $m=M / M_{\odot}$ is the WD's mass in CVs $M$, where the mean mass of these sources is $\mathrm{M}_{\mathrm{CV}}=0.822 \mathrm{M} \odot, R_{9}$ is the $\mathrm{WD}$ radius in units of $10^{9} \mathrm{~cm}, \phi$ is a parameter which is estimated to be $\sim 0.5$ for disc accretion, and $\dot{M}_{16}$ is the accretion rate in units of $10^{16} \mathrm{gs}^{-1}$. The concept is displayed pictorially in Fig. 2, which shows clearly two peaks (bi-modal) Gaussian distributions for most of the CVs with magnetic fields in the range $(0.60-11.20) \times 10^{3}$ G. We fit the Gauss function (same as Eq. (2)) to this distribution. A full summary of the main fitting results is given in Table 3.

Another interesting point is to investigate the correlation between the magnetic fields of MSPs with bottom fields of $\mathrm{CVs}$, which can be derived according to the conservation of the magnetic flux, in other words, study the magnetic fields that produced by AIC. Let's start, then, with the conservation of the magnetic flux

$$
B_{N S}=B_{f, W D} \times\left(\frac{R_{W D}}{R_{N S}}\right)^{2}
$$

As an example of a typical NS we suggest to adopt a radius $\left(R_{N S}\right)$ of about $15 \times 10^{5} \mathrm{~cm}$, where $\alpha$ can be expresses as $\left(R_{W D} / R_{N S}\right)$, subsequently $\alpha^{2} \sim 10^{5.5-6}$, and $B_{f} \sim 10^{3} \mathrm{G}$ in CVs. As a result, one arrives at an expression for the minimum value of magnetic field in NSs equals to:

$$
B_{N S} \sim 10^{8.5-9} G
$$

which is somehow similar to the observational values of MSPs.

To study the contribution of magnetic field of CVs on the MSPs population, we produced a histogram of the total sample of MSPs (Fig. 3), which shows a clear Gaussian distribution for most of the objects, while the observed sample shows two peaks and a small fraction of these systems have intersection. We note that the pinnacle of derived histogram is much higher than in observed, this is due to the sensitivity of the radio observation process for MSPs. Since all pulsar surveys have some limiting flux density (Lorimer (2008)). However, new data acquisition systems provide significantly improved sensitivity to MSPs and are being used to resurvey the sky. Therefore the observed numbers of MSPs of magnetic field are relatively high compare with CVs population. It is noteworthy to mention here that we also found that $\sim 15 \%$ of MSPs could potentially be formed from CVs via AIC process. The peak of the distribution for derived sample occurs at $\sim \times 10^{9.4} \mathrm{G}$ comparing with $\sim \times 10^{8.5} \mathrm{G}$ for the observed one. Furthermore, very high magnetic field Polar systems (like AM Her) are not represented in this figure.

\subsection{Spin period}

It is well established that the NS in binary MSP systems forms first, descending from the initially more massive of the two binary stellar components. The NS is subsequently spun-up to a high spin frequency via accretion of mass and angular momentum once the secondary star evolves (For a more detailed review, see i.e. Alpar et al. (1982); Bhattacharva and van den Heuvel (1991); Tauris et al. (2000); Liu and Chen (2011); Tauris (2011)) In this section we will determine the spin period of MSPs originated from WDs, the process begins with simple Keplerian frequency, the angular velocity of the NS is equal to the Keplerian 
angular velocity of the magnetosphere, at roughly the Alfvén surface,

$$
v_{K} \propto R_{N S}^{-3 / 2} \rightarrow P_{M S P} \sim R_{N S}^{3 / 2}
$$

from which we obtain $P_{M S P}$ as a function of $P_{W D}$

$$
P_{M S P} \sim P_{W D, \min }\left(\frac{R_{N S}}{R_{W D}}\right)^{3 / 2}
$$

where the minimum spin period of the standard WD (de Jager et al. (1994); Middleton et al. (2011)) taking value $\sim 30 \mathrm{~s}, R_{N S}=10 \mathrm{~km}$ and $R_{W D}=1000 \mathrm{~km}$ (see Eqn 1), giving

$$
P_{M S P} \sim 1 m s
$$

Note that the estimated spin periods are not far from the true spin periods of the MSPs. This clearly supports the view that the AIC effect play an important role in deriving spin periods for MSPs.

Fig. 4 shows the observed and derived spin period distributions of MSPs. It can be found that most of derived values centered at $0.011 \mathrm{~s}$ and ranging from 0.011 to $52.8 \mathrm{~s}$. As for the observed MSPs, shows relatively Gaussian and regular distributions for most objects, centered at $4.4 \mathrm{~ms}$ and ranging from $1.4 \mathrm{~ms}$ to $19.5 \mathrm{~s}$. According to these distributions, the ratio of MSPs originated from CVs is about $\sim 10 \%$. This result agrees with some theoretical predictions such as those by Warner (1995) and Warner and Woudt (2002).

\subsection{Mass}

Most of the stellar binary systems consist of two well-separated components, with a little or no interaction beyond their mutual gravitational attraction. However, in close binary systems tidal effects become important, leading to a circularization of the orbit, and spinning up the star's rotational speeds so that they become synchronous with the orbital motion.

The current edition (7.16) 1 of the Catalogue of Cataclysmic Binaries Ritter and Kolb (2011) contains a wide variety and natures of sources such as $880 \mathrm{CVs}, 98 \mathrm{LMXBs}$, and 319 related objects. Among them we have just 18 massive $\mathrm{CVs}$ in the range $1.1-1.3 \mathrm{M}_{\odot}$, which is

\footnotetext{
${ }^{1}$ http://www.mpa-garching.mpg.de/RKcat/
}

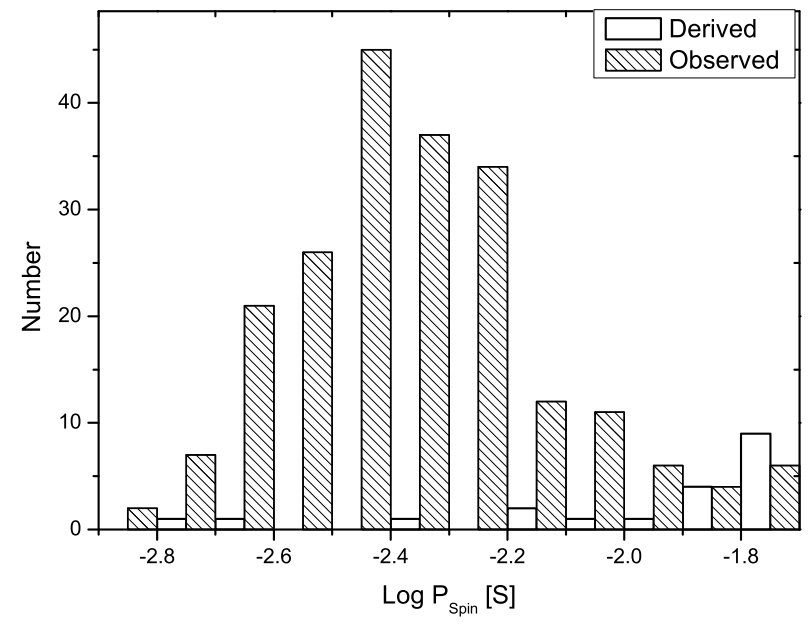

Fig. 4.- The distribution of MSPs on the base of spin-period; Observed (shaded histogram) and derived (solid histogram). The data for MSPs were taken from the ATNF catalogue (Manchester et al. 2005)

considered a relatively small number. A summary of the known properties of these systems is given in table (1). If matter is accreted at a rate of $\dot{\mathrm{M}} \sim 10^{-9} \mathrm{M}_{\odot} / \mathrm{yr}$ to $10^{-8} \mathrm{M}_{\odot} / \mathrm{yr}$ (Zhang and Kojima (2006)), and the total mass accreted exceeds a critical value $\Delta \mathrm{M}_{\text {crit }} \sim 0.1-$ $0.2 \mathrm{M}_{\odot}$ with $10^{9}$ yrs, then the massive $\mathrm{CV}$ will be recycled to become a MSP after the mass reaches the Chandrasekhar limit. It is worth noting here that the mass of the newly formed object will be below the maximum mass for MSP (van den Heuvel (2004)). This provides evidence for the AIC scenario in massive CVs and evolutionary hypotheses of MSP birthrate. In sum up, whether a CV might possibly do this will depends on: (i) the mass of the WD in the system, and (ii) the mass transfer rate that the donor star may supply, because we know that at low mass transfer rates one only gets $\mathrm{SN}$ explosions Type Ia, and the WD will never grow to become a NS.

It should be noticed that the AIC process leads to MSP with mass less than Chandrasekhar limit. When a WD gains mass by accreting matter from its companion, its binding energy also increases, this effect is significant in the estimation of the amount of mass accretion. However, unfortunately most of the studies argued that the binding energy of the NS is not commonly considered 
Table 1: List of physical properties for the all massive CVs $\left(M \geq 1.0 M_{\odot}\right) *$

\begin{tabular}{lcccl}
\hline \hline Name & $M / M_{\odot}$ & $P_{\text {Orb }}(\mathrm{D})$ & $R_{\text {Derived }}\left(10^{9} \mathrm{~cm}\right)$ & $B_{\text {Derievd }}\left(10^{3} \mathrm{G}\right)$ \\
\hline CAL 83 & 1.3 & 1.04 & 0.76 & 13.95 \\
CI Aql & 1.2 & 0.618 & 0.83 & 12.38 \\
BV Cen & 1.24 & 0.61 & 0.8 & 13 \\
EY Cyg & 1.1 & 0.49 & 0.9 & 10.86 \\
RU Peg & 1.21 & 0.37 & 0.82 & 12.53 \\
QZ Aur & 1.05 & 0.357 & 0.95 & 10.13 \\
MU Cen & 1.2 & 0.342 & 0.83 & 12.38 \\
BF Eri & 1.28 & 0.27 & 0.78 & 13.63 \\
VY Scl & 1.22 & 0.232 & 0.81 & 12.69 \\
RX And & 1.14 & 0.209 & 0.87 & 11.46 \\
SS Aur & 1.08 & 0.182 & 0.92 & 10.57 \\
BD Pav & 1.15 & 0.179 & 0.86 & 11.61 \\
U Gem & 1.2 & 0.176 & 0.83 & 12.38 \\
V603 Aql & 1.2 & 0.138 & 0.83 & 12.38 \\
WW Hor & 1.1 & 0.08 & 0.9 & 10.86 \\
CU Vel & 1.23 & 0.078 & 0.81 & 12.84 \\
DP Leo & 1.2 & 0.062 & 0.83 & 12.38 \\
\hline
\end{tabular}

*The masses and orbital periods were taken from Ritter and Kolb (2011), while B and R are determined by the condition that the magnetosphere equal to the WD radius (Zhang et al. 2009). The model-dependent parameters are $\phi=0.5$ and $\dot{M}=10^{16} \mathrm{gs}^{-1}$, (see the text).

Table 2: The fitting results of distributions for mass of Cvs.

\begin{tabular}{|c|c|c|c|c|c|c|c|}
\hline quantity & $y_{0}$ & $x_{c}$ & $w$ & $\bar{A}$ & $\sigma$ & $\chi^{2} /$ Dof & 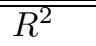 \\
\hline $\mathrm{M}_{C V s}$ & $0.53 \pm 0.64$ & $0.79 \pm 0.012$ & $0.40 \pm 0.03$ & $12.30 \pm 0.92$ & 0.20 & 3.311 & 0.955 \\
\hline
\end{tabular}

Table 3: The fitting results of distributions for derived magnetic field strength in CVs .

\begin{tabular}{lcccccccc}
\hline quantity & $y_{0}$ & $x_{0}$ & $x_{1}$ & $w_{0}$ & $w_{0}$ & $A$ & $B$ & $R^{2}$ \\
\hline & & & & & & & & \\
$B_{\text {Derived }}$ & $-0.05 \pm 0.004$ & $3.93 \pm 0.04$ & $3.41 \pm 0.08$ & $0.186 \pm 0.008$ & $0.37 \pm 0.003$ & $14.42 \pm 4.57$ & $5.45 \pm 1.35$ & 0.942 \\
\hline \hline
\end{tabular}


(Bagchi (2011)).

\subsection{Orbital periods and eccentricity}

We now believe that in the case of spherical $\mathrm{SN}$ explosions in which $\mathrm{C}-\mathrm{O}$ WD (at low mass transfer rates) produced impart significant kicks to the companion that make their survival prospects within binary systems in a long orbital period or bleak. The asymmetric mass loss during the AIC process is expected to provide kick velocities of about $50 \mathrm{kms}^{-1}$ or less (Gothoskar and Gupta $(2000)$ ). Consequently, the AIC produces very low eccentricities $(e<0.1)$. When the natal MSPs receive a relatively strong kick $\left(>100 \mathrm{kms}^{-1}\right)$, the AIC channel produces eccentric binary MSPs in the Galaxy. Such a kick seems to be highly unlikely in the AIC process, hence the probability of forming eccentric binary MSPs via the AIC channel can be ruled out. Even if a high kick is allowed, the AIC channel cannot produce eccentric with an orbital period of $\geq 70 \mathrm{~d}$ Chen et al. (2011).

In sum up, the high-mass binary systems tend to have highly eccentric orbits, while the low-mass binaries have smaller eccentricities. The origin of MSPs in low-mass binaries could be due to either AIC or supernova explosion. If MSPs are formed by the AIC process, their average velocity would be much lower than that of normal pulsars. Further work will certainly clarify this issue as known sources are better characterized.

\section{Summary and Conclusions}

We have studied the possible AIC scenario in massive CVs, through simple calculations of some physical characteristics such as magnetic field, spin period and mass. The basic information that goes into the calculation can be summarized as follows

1. CVs would be invoked via the capability of producing a significant portion of the MSPs population through the AIC process, a regime which otherwise may be unattainable by the normal channels that produce NSs (i. e. long orbital periods for half of the MSPs and the formation of single MSPs) in order to reduce the gap between standard LMXB evolution and the observed population of MSPs.
2. We find that the quantitative implications of our calculations are that, we can estimate the expected magnetic field strength in the observed population of MSPs which could be contributed from CVs is about $\sim 15 \%$. While it turns out that the contribution in spin period is about $\sim 10 \%$. It would be very interesting though, if observations would yield either a very slowly spinning (few hundred ms) pulsar associated with a very low magnetic field, or a high magnetic field MSP with a very rapid spin. (However, the latter kind of pulsars might be unlikely to be detected given that its very strong magnetic dipole radiation would slow down its spin rate within a few Myrs).

3. We compute the bottom magnetic field in CV samples, with a discussion on the implications of the model in Zhang et al. (2009). We extend this model to conditions appropriate to accreting WDs. Thus the model predicts that the magnetic polar cap widens as material is accreted with the field being advected towards the equatorial regions by the ensuing hydrodynamic flow.

4. On the basis of our results we conclude that, it is possible to make comparisons about the relative importance of spin periods, magnetic field and some other parameters in CV population, that potentially produced via AIC process about their aggregate impact in the observed population of MSPs.

5. The mass of MSPs which produced via AIC process is less than Chandrasekhar limit, because at least $\sim 10 \%$ of the original mass goes to the binding energy of the NS.

6. We further find that the predictions of some parameters after AIC process for the average levels are consistent with the observed population (such as spin period, magnetic field and mass). Future work will go steps further, and consider other observable quantities such as orbital period, eccentricity and mass ratio (q) for MSP populations using the more extensive data set now available from Ritter and Kolb (2011) and other sources.

Ultimately, the critically unique analysis will be the cross correlation with the CVs, AIC pro- 
cess and MSPs, which is well beyond the scope of this letter. Our main conclusion is that the simple calculations presented herein, based on the standard relationships between mass, magnetic field, spin and orbital periods, required to support the more sophisticated treatments of the problem, and suggests that how the AIC contributes to the observed population of MSPs.

\section{Acknowledgements}

The research presented here has made extensive use of the 2012 version of the ATNF Pulsar Catalogue (Manchester et al. 2005). Dr. AlWardat would like to thank Max Planck Institute for Astrophysics-Garching for the hosting funded by the Deutsche Forschungsgemeinschaft (DFG) during the writing of this work. This research has been supported by NSFC (No.10773034) and National Basic Research Program of China (2012CB821800). We thank the anonymous referee for a careful reading of our manuscript and for numerous useful comments.

\section{REFERENCES}

Alpar, M.A., Cheng, A.F., Ruderman, M.A., Shaham, J.: Nature 300, 728 (1982). doi:10.1038/300728a0

Bagchi, M.: MNRAS 413, 47 (2011). 1102.2912. doi:10.1111/j.1745-3933.2011.01030.x

Bhattacharya, D., van den Heuvel, E.P.J.: Phys. Rep. 203, 1 (1991). doi:10.1016/03701573(91)90064-S

Chandrasekhar, S.: An Introduction to the Study of Stellar Structure, (1939)

Chen, W.-C., Liu, X.-W., Xu, R.-X., Li, X.D.: MNRAS 410, 1441 (2011). 1008.2130. doi:10.1111/j.1365-2966.2010.17512.x

de Jager, O.C., Meintjes, P.J., O’Donoghue, D., Robinson, E.L.: MNRAS 267, 577 (1994)

Ferrario, L., Wickramasinghe, D.T.: In: Y.F. Yuan, X.-D. Li, \& D. Lai (ed.) Astrophysics of Compact Objects. American Institute of Physics Conference Series, vol. 968, p. 194 (2008a). doi:10.1063/1.2840395
Ferrario, L., Wickramasinghe, D.T.: In: Y.F. Yuan, X.-D. Li, \& D. Lai (ed.) Astrophysics of Compact Objects. American Institute of Physics Conference Series, vol. 968, p. 188 (2008b). doi:10.1063/1.2840393

Gothoskar, P., Gupta, Y.: ApJ 531, 345 (2000). doi:10.1086/308450

Hurley, J.R., Tout, C.A., Wickramasinghe, D.T., Ferrario, L., Kiel, P.D.: MNRAS 402, 1437 (2010). doi:10.1111/j.1365-2966.2009.15988.x

Isern, J., Hernanz, M.: Mem. Soc. Astron. Italiana 65, 339 (1994)

Ivanova, N., Taam, R.E.: ApJ 599, 516 (2003). arXiv:astro-ph/0308361. doi:10.1086/379192

King, A.R., Kolb, U., de Kool, M., Ritter, H.: MNRAS 269, 907 (1994)

Knigge, C., Baraffe, I., Patterson, J.: ApJS 194, 28 (2011). 1102.2440. doi:10.1088/0067$0049 / 194 / 2 / 28$

Li, X.: Science in China G: Physics and Astronomy 53, 9 (2010). doi:10.1007/s11433-0100034-0

Liu, W.-M., Chen, W.-C.: MNRAS 416 , 2285 (2011). 1106.1567. doi:10.1111/j.13652966.2011.19202.x

Lorimer, D.R.: Living Reviews in Relativity 11, 8 (2008). 0811.0762

Manchester, R.N., Hobbs, G.B., Teoh, A., Hobbs, M.: AJ 129, 1993 (2005). doi:10.1086/428488

Middleton, M.J., Cackett, E.M., Shaw, C., Ramsay, G., Roberts, T.P., Wheatley, P.J.: $\quad$ MNRAS, 1594 (2011). 1108.1105. doi:10.1111/j.1365-2966.2011.19696.x

Nomoto, K.: ApJ 277, 791 (1984). doi:10.1086/161749

Nomoto, K.: ApJ 322, $206 \quad$ (1987). doi:10.1086/165716

Qian, S.-B., Liu, L., Liao, W.-P., Li, L.-J., Zhu, L.-Y., Dai, Z.-B., He, J.-J., Zhao, E.-G., Zhang, J., Li, K.: MNRAS 414, 16 (2011). 1103.2005. doi:10.1111/j.1745-3933.2011.01045.x 
Rappaport, S., Verbunt, F., Joss, P.C.: ApJ 275, 713 (1983). doi:10.1086/161569

Ritter, H., Kolb, U.: VizieR Online Data Catalog 1, 2018 (2011)

Romani, R.W.: Nature 347, 741 (1990). doi:10.1038/347741a0

Spruit, H.C., Ritter, H.: A\&A 124, 267 (1983)

Taam, R.E., van den Heuvel, E.P.J.: ApJ 305, 235 (1986). doi:10.1086/164243

Taani, A., Zhang, C.M., Al-Wardat, I., Zhao, Y.H.: Astronomische Nachrichten 333, 8 (2012). 1112.1312v1. doi:10.1002/asna.201111611

Tauris, T.M.: ArXiv e-prints (2011). 1106.0897

Tauris, T.M., van den Heuvel, E.P.J., Savonije, G.J.: ApJ 530, 93 (2000). arXiv:astroph/0001013. doi:10.1086/312496

van den Heuvel, E.P.J.: Journal of Astrophysics and Astronomy 16, 255 (1995)

van den Heuvel, E.P.J.: Science 303, 1143 (2004)

Wang, J., Zhang, C.M., Zhao, Y.H., Kojima, Y., Yin, H.X., Song, L.M.: A\&A 526, 88 (2011). 1011.5013. doi:10.1051/0004-6361/201015190

Warner, B.: Cambridge Astrophysics Series 28 (1995)

Warner, B., Woudt, P.A.: In: B. T. Gänsicke, K. Beuermann, \& K. Reinsch (ed.) The Physics of Cataclysmic Variables and Related Objects. Astronomical Society of the Pacific Conference Series, vol. 261, p. 406 (2002). arXiv:astroph/0109397

Wickramasinghe, D.T., Ferrario, L.: MNRAS 356, 1576 (2005). doi:10.1111/j.13652966.2004.08603.x

Wickramasinghe, D.T., Hurley, J.R., Ferrario, L., Tout, C.A., Kiel, P.D.: Journal of Physics Conference Series 172(1), 012037 (2009). doi:10.1088/1742-6596/172/1/012037

Wood, M.A.: In: D. Koester \& K. Werner (ed.) White Dwarfs. Lecture Notes in Physics, Berlin Springer Verlag, vol. 443, p. 41 (1995)
Zhang, C.M., Kojima, Y.: MNRAS 366, 137 (2006). doi:10.1111/j.1365-2966.2005.09802.x

Zhang, C.M., Wickramasinghe, D.T., Ferrario, L.: MNRAS 397, 2208 (2009). 0905.4829. doi:10.1111/j.1365-2966.2009.15154.x

This 2-column preprint was prepared with the AAS LATEX macros v5.2. 